\title{
Meurtre, souillure et cannibalisme dans une chefferie mélanésienne maré (Iles Loyauté)
}

Murder, impurity and cannibalism in the Melanesian chiefdom of Maré (Loyalty Islands

Charles Illouz

\section{OpenEdition}

\section{Journals}

Édition électronique

URL : http://journals.openedition.org/span/1483

DOI : $10.4000 /$ span. 1483

ISSN : 2268-1558

Éditeur

École pratique des hautes études. Sciences humaines

Édition imprimée

Date de publication : 1 août 1996

Pagination : $57-76$

ISSN : 0294-7080

Référence électronique

Charles Illouz, « Meurtre, souillure et cannibalisme dans une chefferie mélanésienne maré (Iles Loyauté) ", Systèmes de pensée en Afrique noire [En ligne], 14 | 1996, mis en ligne le 17 avril 2014 consulté le 11 octobre 2020. URL : http://journals.openedition.org/span/1483 ; DOI : https://doi.org/ 10.4000/span.1483 


\section{MEURTRE, SOUILLURE ET CANNIBALISME DANS UNE CHEFFERIE MÉLANÉSIENNE MARÉ (Iles Loyauté)}

\section{par}

\section{Charles Illouz}

Dans une société guerrière comme celle de Maré où les conflits violents se sont multipliés jusqu'à la fin du XIX ${ }^{\mathrm{e}}$ siècle, bien après la prise de possession de l'archipel des Loyauté par la France, on s'attendrait à trouver dans l'ensemble des dispositifs d'intégration sociale un statut très marqué pour le tueur d'ennemis. Un tel statut n'est pourtant pas observable, du moins selon la corrélation qui se fait jour ailleurs entre le guerrier, agent individuel du meurtre, et sa victime, vis-à-vis de laquelle il occuperait une place réversible. Bien des sociétés, en effet, tiennent les tueurs à distance tant qu'une manipulation symbolique n'est pas venue les libérer de la souillure que le sang versé a jetée sur eux ${ }^{1}$. Sous cet angle, l'enquête sur le terrain maréen laisse apparaître un hiatus.

1 Plusieurs auteurs mentionnent ces pratiques en Océanie. M. Godelier rapporte que * les Baruya, quand ils avaient tué un ennemi au combat, plongeaient leurs armes dans le sang qui coulait de ses blessures, et ils poussaient leur cri de victoire. Ou bien ils sacrifiaient un guerrier capturé vivant, en lui perçant le corps avec un couteau sacré en bambou, et ils se peignaient le corps du sang qui jaillissait. Puis ils l'achevaient en lui arrachant le foie, qu'ils cuisaient et mangeaient. Après quoi ils ne pouvaient 
C'est que, dans la chefferie maré où toute l'organisation vise à la constitution et au maintien de la personne du chef, le meurtre, comme toutes les atteintes faites au corps, ne peut relever completement de la compétence du guerrier. Il existe néanmoins un rite de passage lors duquel les jeunes gens, déjà instruits au Tacaer(e), la grande case des garçons et des jeunes guerriers, sont flagellés jusqu'au sang (camethot) par leurs aînés immédiats en manière d'introduction dans la troupe militaire (eat) où ils sauront s'illustrer. Mais il s'agit là d'une initiation collective qui prévoit mais ne sanctionne pas le meurtre guerrier.

Dans la rhétorique de guerre, deux expressions mettent le sang au premier plan: hmu-me-dra "le casse-tête sanglant", qui signifie "offensive", l'initiative dans l'attaque, et thuba-dra "le prix du sang " payé aux alliés dans la guerre pour les morts qu'ils y ont laissés. Que se passe-t-il entre ces «introduction et conclusion du sang " ? En quels termes dit-on le procès meurtrier lui-même et la sanction de l'homicide? Entre cette " offensive " et ce " règlement ", nous verrons que les protocoles du sang s'effacent devant une autre instance meurtrière qui procède en dernier lieu de la définition même de la chefferie.

La réflexion à laquelle nous engageaient $M$. Detienne et M. Cartry sera donc abordée, non à partir du sujet social « ordinaire ", mais a partir de la personne du chef sur laquelle se focalisent les institutions maréennes qui traitent aussi du meurtre. C'est sous cet angle qu'on voit formellement apparaitre « du chef » selon le double

\footnotetext{
regagner leur village et reprendre une vie normale sans s'être lavé et purifié rituellement le corps de toute trace de sang... (1982: 101). Toujours sur le terrain néoguinéen, B. Juillerat rapporte que chez les Yafar * tout meurtrier novice, s'il restait sain de corps, devenait fragile par le "mûrissement" momentané de son "moi", et devait en conséquence se protéger contre toute attaque de sorcellerie aysiri, et même contre les piqûres d'insectes et de plantes urticantes. Cependant, par la répétition de l'homicide, le combattant peu à peu s'immunisait au processus de mûrissement... Il semble qu'on ait là la rencontre d'un double processus contradictoire : celui du contact avec le sang entraînant le "moi" sur la voie du pourrissement, et celui de l'assumation d'un acte de courage qui, à l'inverse, affermit l'identité du sujet parvenant à dépasser sa peur» (1986: 469).
} 
principe de l'efficience meurtrière et de son anticipation protectrice dans le champ omniprésent de la souillure.

Le chef apparaît dans la relation qu'il entretient avec un certain nombre de personnages. Ceux-là ont pour la plupart le pouvoir de se substituer à lui, selon les occasions, pour prendre la parole, acte reconducteur, on le verra, de l'organisation sociale dans les multiples rapports que nouent, dans leur ensemble numérique, les « sujets » de la chefferie.

Une catégorie de ces factotum du chef revêt l'apparence d'une " entité ", conçue en l'espèce de plusieurs personnes (représentant chacune l'ensemble d'un lignage) titulaires de compétences redoutables et complémentaires. Il s'agit de l'acania, textuellement le « maître du mal ". L'évidence du " mal" dont l'acania possède la maîtrise s'impose sous la forme d'une "magie " meurtrière qui se pratique à partir du corps du chef. Cette "magie ", le kaze, est réputée avoir été amenée par des gens de Lifou, île voisine au nord-ouest de Maré. Si l'introduction des groupes acania dans les chefferies constituées est toujours présentée de manière à en souligner le caractère étranger, c'est surtout parce que les acania conservent des liens qui passent outre l'appartenance stricte à la chefferie d'accueil. On raconte aussi en effet l'arrivée de ceux qui furent "placés comme chefs" sans dégager la permanence, en ce qui les concerne, de ce caractère "étranger ». Ce point permettra d'éclairer l'ambivalence par laquelle se constitue la fonction de l'acania dans la chefferie (padoku). Toutes les venues d'acania sont responsables d'un fort infléchissement de l'organisation politique de la chefferie.

Les détenteurs de kaze s'imposent comme les praticiens de la mise à mort, même si le meurtre est effectivement exécuté par un quidam guerrier. On verra en effet que le meurtre est toujours imputé au kaze, lequel soumet en quelque sorte la victime au casse-tête ou à la lance du guerrier. De même, toute mort dans la " tribu " pourra être interprétee de cette façon si l'on présume que le défunt a perdu son foie ; nous verrons plus bas pourquoi cet organe.

En cela, le kaze est réputé tuer à distance, et le traitement ultérieur du cadavre en restitue l'évidence. Le meurtre, et la souillure qui l'accompagne, reposent ainsi totalement sur le « maître du mal ». 
Parmi les personnages attachés à la demeure du chef, le plus " inoffensif " est sans doute le sinemeneng, et sa suite, "serviteur ", « chambellan " du chef. Il pourvoit à sa nourriture comme à tous ses besoins domestiques et le soigne ou plutôt prévient la maladie en lui proposant des remedes (serei(e)). Il agit ouvertement et son action est absolument distincte de celle des maîtres du mal qu'il croise dans l'enceinte de la résidence.

Chez les maitres du mal, celui qui est dit core'ma " dos-demaison", ou gorehnameneng "enceinte-de-demeure" (goron: "déployer sur le pourtour "), et dont on dit qu'il est la " sentinelle ", défend les accès de la résidence du chef, et ceux de son territoire de suzeraineté. C'est lui qui détient le kaze dont il peut " frapper " toute personne tombée en disgrâce ${ }^{2}$.

Kaze signifie " cadavre ", à un stade particulier toutefois. En effet, le cadavre ordinaire, le mort, se dit tango ou net. Le kaze est le cadavre qui, ayant reçu une sépulture, sera détourné de sa destination funebre. Pour cela il faut avoir rendu la dépouille du défunt aux maternels, seuls habilités à l'ensevelir, ou autrefois à déposer le corps dans une grotte ${ }^{3}$. Ces derniers ont préalablement pris le repas de deuil et ont reçu de grandes quantités de présents ( $c a$ ue).

L'intervention des maternels permet la circulation du nen(e), l' "énergie " dont le défunt a besoin dans sa migration post-mortem. C'est donc ainsi chargé de nen(e $)^{4}$ que le cadavre pourra servir à la fabrication du kaze. Le terme kaze est d'ailleurs une synecdoque pour

${ }^{2}$ La personne frappée du kaze est souvent invectivée publiquement pour toutes sortes de raison et sa mort, qui peut survenir n'importe quand, est nécessairement l'auvre du kaze.

3 En fait les hnakasese * ex-commensaux * disposent aussi de ce privilège et en disposent souvent avant les maternels. L'institution qui fait des hnakasese une sorte de doublure des maternels s'explique historiquement par un lien matrimonial dont ils se sont défaits mais qui est rappelé dans toutes les occasions qui exigent la présence des maternels. Il est intéressant de préciser pour notre sujet que les hnakasese ne peuvent jamais se nuire et qu'ils ne peuvent donc pas être réciproquement vulnérables au kaze dont ils usent.

4 Au nen(e) maré correspond le men(e) lifou et le mana polynésien. Nene signifie également * mère, maman *. Cette assimilation lexicale répond à l'influence décisive des maternels sur la circulation de l' " énergie ". 
du-re-kaze "os de cadavre ". Il est aussi désigné par l'équivalent $d u$-re-coco « os de coq " parce que le chef est représenté par un coq (doku drera "chef rouge " par référence à la crête du gallinacé). On verra en quoi la figure du coq n'est pas étrangère à la nature du lien qui unit le chef à son maître du mal.

Le kaze est ainsi de l'os de chef chargé de nen(e). Le premier acte de l'acania core'ma consiste donc à chercher les sépultures de chefs au cours de déambulations nocturnes afin d'y dérober les précieux os, ingrédients majeurs du paquet magique ficelé appelé kaze.

Or, ce cadavre de chef est rendu introuvable, ou presque, par les soins d'un autre maître du mal, l'acania me kug(o), celui « qui est sale, souille ". Très étroitement attaché à la personne du chef, le maître du mal-souillé est ainsi nommé parce qu'il doit en absorber les excréments et, d'une manière générale, tout ce que libère le corps du chef ${ }^{5}$. Ainsi, lorsque ce dernier est blessé ou malade, il consomme ses crachats, son pus ou son sang. "Il touche les saletés du chef " $(c i$ $r u(e) k u g(o) o d o k u$ ), il est "celui à qui on jette le paquet " (la "poubelle") (ngom cili ke-re-ael). Lui seul supporte le contact du chef dont il mange aussi les restes du repas; le chef en retour est seul a supporter son contact. Cet escamotage alimentaire de tous les déchets du chef ira, à la mort de ce dernier, jusqu'à la consommation des chairs putrescentes de son cadavre. L'acania me kug(o) «boit» le cadavre avec un roseau tout au long de sa décomposition (ci kua bi re tango ne guashow). La chose est pratiquée dans la grotte secrète où il a caché le cadavre (ulan) dont il s'est emparé. Selon l'expression française il est presque littéralement le " croque-mort " du chef.

Depuis que les évangélistes ont convaincu les insulaires d'ensevelir leurs morts, le maître du mal-souillé est enterré à l'extérieur des cimetières. De même nul n'osera manger dans une assiette qu'il a touchée. Constitué sur l'excrément et le cadavre du chef, le maître du mal devient ainsi son double mortifere.

Notons enfin que la tête de la tortue est exclusivement réservée à ce personnage, ce dont nous reparlerons.

${ }^{5}$ Hormis les cheveux et les phanères. 
Comme double mortifère du chef, il porte aussi le titre de ce dernier. Dans les chefferies de l'est, on le nomme acania doku, c'està-dire " maître du mal chef ». Or, lorsqu'on passe à l'ouest, dans celle de Guahma, (la plus importante), on observe que la fonction s'est clivée de manière à laisser apparaître un porteur de titre "maître du malchef " distinct du maître du mal-souillé. Le clivage d'une même figure est d'autant plus remarquable que les deux " maîtres du mal " portent le même nom de personne, Waraw(i). L'identité que le maître du mal-chef entretient par le titre avec le chef d'une part, et avec le maître du mal-souillé par scissiparité d'autre part, se traduit par le fait que le maître du mal-souillé traitera sa " moitié " comme un chef, soit en absorbant ses excréments et son cadavre le jour venu. Chef et maître du mal-chef se déversent tous deux dans le même maître du mal-souillé.

Cet avatar abouti de la fonction traduit en dernier lieu l'autorité politique grandissante de l'acania. Le maître du mal phagocyte le chef et opère identiquement, peut-être même plus complètement, à l'autre « extrémité " de la structure de la chefferie.

Ein effet, le chef - théoriquement l'aîné de la lignée dans le lignage aîné (retok) - voit son statut défini par celui du eteshet - théoriquement le dernier-né de la lignée dans le lignage du dernier-né. La place du " premier-né " (retok) est définie par rapport à celle du « dernier-né " (eteshet), créant ainsi l'espace institutionnel où peuvent s'inscrire tous les lignages « cadets " (re-celua). Institutionnel, en effet, car le cadre de symétrie "premier-né/dernier-né » s'appuie sur la délégation de parole du "premier " au « dernier ». Cette parole est fondatrice de l'ordre et reconductrice de l'ordonnance de la société, nous verrons de quelle façon. Le chef premier-né ne parle donc que par la bouche du dernier-né ${ }^{6}$.

${ }^{6}$ Remarquons que le terme eteshet signifie stricto sensu * vieille femme * et qu'il est appliqué aux vieux hommes du lignage qui ont rang de dernier-né. Cette confusion terminologique qu'on paraît ignorer au plan de l'institution se retrouvera ailleurs et nous permettra alors de soulever le rapport qui détermine cette inversion des sexes. Pour lors nous traduisons eteshet par * vieux derniers-nés *. 
Outre cette délégation de parole, qui tombe aussi nous l'avons vu sous la compétence du maître du mal en certaines occasions, le "dernier-né * détient, depuis la fondation de la "chefferie ", un "petit-panier » (waceng) renfermant les magies guerrières de son clan (guhnameneng), celles donc initialement de la chefferie. La venue des acania frappe en quelque sorte cette magie d'obsolescence. Ils proposent en effet une magie infiniment plus « énergique " puisque issue du corps même des chefs qu'ils prétendent, pour les mêmes raisons, soustraire à de telles magies.

Le maître du mal procède donc par « recopiage » de la structure initiale dans l'espace exclusif d'une compétence supérieure à fabriquer de la mort pour le raffermissement de la chefferie. Si son autorité sur le contrôle social grandit au détriment du chef et de son dernier-né, c'est qu'il opere dans le champ structurel global des institutions de la chefferie avec des instruments que partagent seulement les autres maîtres du mal de l'île.

On ne peut donc pas dire que la chefferie maré commence là où il $\mathrm{y}$ a du chef, mais véritablement là où une relation qui dépasse la séparation des chefferies - celle des acania d'une chefferie à l'autre - requiert le prétexte du maintien des chefs. Ce compromis se révèle clairement par le titre de " chef » qui échoit secondairement au maître du mal-souillé.

Ce glissement d'autorité explique le déplacement opéré du point de vue symbolique du sang vers les feces, substance constitutive du maître du mal dans sa relation au chef. En effet, nous avons signalé plus haut que les représentations guerrières n'accordent au sang qu'un espace rhétorique: à l'initial du «mouvement» guerrier par le hmu-me-dra, le * casse-tête sanglant », soit l'offensive, et au final par le thuba-dra, le « règlement du sang "; l'opération meurtrière elle-même relevant en fin de compte de la manipulation du kaze, os de chef dégagé des chairs putrescentes lesquelles sont absorbées, comme les excréments, par le maître du mal. Comme si le sang qui aurait été répandu sans l'intervention du maître du mal, donc par l'efficience du "petit-panier » (waceng) du dernier-né (eteshet), cédait la place aux excréments mortiferres dont nous devons à présent chercher de quelle manière ils sont “ répandus " lors du meurtre. 
Pour percevoir précisément les modalités d'un tel déplacement et introduire une autre notion déterminante dans le glissement de l'autorité vers le maître du mal, celle du cannibalisme, il est utile d'exposer brièvement les cycles cérémoniels de prestations agricoles qui rendent compte de l'ensemble des rapports sociaux au sein de la chefferie. On comprendra ainsi qu'à ce super meurtrier correspond une super victime : le chef lui-même, dont le corps, philtre mortifère du maître du mal, circonscrit de son vivant l'ensemble des sujets de la chefferie.

C'est au début du mois de février qu'on prépare et qu'on accomplit le rekoko, "griller l'igname ». Il s'agit pour le chef "premier-né " de préparer et d'offrir au dernier-né (eteshet) des igmames nouvelles ficelées dans un paquet de feuilles de bananiers dit aebon, "paquet-qui-sent ", cuit au four de pierres sans condiments ni lait de coco. Il consommera l'offrande avec les derniers-nés de ses enfants et de ses petits enfants. Après seulement, tout le monde pourra manger les ignames nouvelles cuites de la même façon, mais cette fois avec du poisson.

Ce poisson est pris lors de grandes pêches collectives dites therebon, "chercher-ce-qui-sent ". Dès la pêche terminée, la préparation du poisson offre une réponse à la question posée par une telle désignation. Le poisson est éviscéré et les intestins sont soigneusement vidés et lavés de leur contenu excrémentiel. Les poissons sont alors suspendus aux arbres afin, dit-on, que le vent emporte les humeurs qui y restent attachées. Les intestins seront cuits et servis avant la célébration du rekoko, aux vieux qui ont rang de dernier-né.

Cette offrande préliminaire du aebon au dernier-né est accomplie par le chef mais aussi par tous les " chefs de clan " (tokaguhnameneng) de la chefferie à leur propre dernier-né. La célébration des ignames nouvelles (rekoko) consiste ensuite en invitation réciproque des tribus à des banquets d'ignames et de poissons.

Dans le courant du mois de février a lieu l'offrande du hnadu « ce qui a été cultivé ». Il ne s'agit déjà plus d'ignames nouvelles. Le cadet offre une ou plusieurs ignames à son aîné immédiat, lequel en fait autant à son aîné immédiat, et ainsi de suite jusqu'au « chef de clan " (tokaguhnameneng). Chaque lignée remonte ainsi jusqu'à la " tête " du clan en un trajet dit hnaelen, "là où est la tête ". 
Vers la fin février, les "têtes" hnaelen portent les présents d'ignames au "chef ", celui donc qui a rang d'aîné parmi les aînés de chaque clan, d'où le nom pluriel qui le désigne, retok « les-ânés » dont on admet sur place la traduction de « chef ». Le temps de cette prestation est lakodraru, "chemin de nourriture".

En passant de l'offrande du hnadu "ce qui a été cultivé "à celle de lakodraru, on franchit le seuil qui sépare la sphère de la parenté de l'ensemble numérique des " sujets " sociaux de la chefferie en usant de la même terminologie. Les Maréens traduisent re-celua ni retok " les cadets de l'aîné des aînés " par " les sujets du chef ".

La description qui précede a pour but de montrer que l'inventaire exhaustif des « sujets » de la chefferie (padoku) se réalise pour l'essen$\operatorname{tiel}^{7}$ à partir de la production agricole dite khen, laquelle est présentée comme substance de la " parole » des eteshet "vieux derniers-nés " homonymement appelée khen. Cette parole consiste en effet en discours itératifs sur cette ordonnance sociale et sur les protocoles qui y sont attachés.

La parole, que le chef (retok " premier-né ") délègue au dernierné, est donc consubstantielle à l'igname, et le dernier-né dépositaire doit veiller aux conditions de sa reproduction et de sa prospérité. Hypostase de la parole des " vieux derniers-nés ", la production agricole donne lieu plus avant à l'intervention de ces derniers.

Dans le courant du mois de mars, les «vieux derniers-nés » prennent connaissance de l'état des récoltes afin de corroborer l'impression qu'ils ont eu dès la présentation du lakodraru au chef, fin février. Selon leur jugement on organisera ou non une nouvelle prestation d'ignames pour le chef. On réunit alors, si les récoltes sont satisfaisantes, les yewakoko, "pieds d'ignames ", lors d'une fête dite wanimoc, nom de la fauvette (Gerygone flavolateralis flavolateralis). Il s'agit de faire état des surplus afin que le chef et les vieux derniersnés jugent de l'opportunité d'inviter d'autres chefferies à banqueter.

${ }^{7}$ Les maîtres de la mer acacele, * propriétaires * des rivages, apportent du poisson, celui-là même dont on organise la prise (there bon) lors de grandes battues dans les lagons (cece). 
Autrefois, les chefferies ennemies qui concluaient une paix prévoyaient (ekonejew) de s'inviter tous les trois ans à tour de rôle pour une grande fête wanimoc. Ce qui signifie que la paix entre deux chefferies était reconduite une fois tous les six ans sur les surplus de chacune d'elles. On perçoit alors combien pendant les cinq autres années la paix était favorable aux récoltes. Mais si la guerre réduisait notablement les perspectives de surplus, du fait des stratégies d'embuscades larvées, elle promettait aussi des déficits sur l'ensemble des prestations cérémonielles. Répondant alors peu ou prou, selon les moments de l'année, à la subsistance du groupe, les récoltes témoignaient de la fragilité de la parole des vieux derniers-nés.

C'est de cette fragilité que les acania, promoteurs de guerres, se grandissent avec leur kaze. L'avancée de l'autorité du maître du mal correspond donc en premier lieu au recul des fêtes du surplus wanimoc et à une éventuelle précarité du " chemin de nourriture " (lakodraru) due au chef. Or, le chef, " aîné des aînés ", qui par essence focalise la circulation de l'igname, n'aurait plus alors d'existence sociale si le maître du mal n'avait le pouvoir d'en garantir le maintien. Le « chemin de nourriture » (lakodraru) cède la place au puec, dont le sens est celui de "tribut", et dont on peut dire qu'il va, sous la férule du maître du mal, jusqu'à prendre l'aspect d'une extorsion. En effet, lors de la remise du puec dans certaines chefferies, les sujets récalcitrants étaient " remerciés » (shudul) d'un coup de lance afin que leurs chairs accompagnent les ignames présentées. Les prestataires devaient donc passer maîtres dans l'art d'esquiver les jets de lance ${ }^{8}$. Dans cette relation de puec avec ses sujets, le chef est désormais appelé doku (et non plus retok), nom qu'il partage nous l'avons vu avec le maître du mal-souillé, et qui marque la séparation de sa personne d'avec

${ }^{8}$ C'était le cas de ceux qui prétendaient ne devoir que le * chemin de nourriture * (lakodraru), se référant en cela à la parcle des vieux derniers-nés, afin de conserver l'indépendance d'où pourrait se détacher, l'histoire faisant, leur propre * chefferie *. La fuite de ceux qui avaient esquivé le coup de lance du doku a parfois permis la constitution de chefferies rivales. 
l'ensemble de la société à laquelle il ne répond plus en tant qu'* aîné des aînés » mais d'une certaine façon en tant que « roi »".

Dans la chefferie de Guahma, où le maître du mal-souillé s'est clivé pour permettre qu'une "partie de lui-même " soit traitée comme le roi en s'offrant, tel ce dernier, au contact de son "autre partie souillée ", le calendrier cérémoniel en est même définitivement modifié. C'est seulement début mars, après une rapide célébration du rekoko, qu'a lieu la présentation des puec qui, ainsi placée, recouvre celles des hnadu, du lakodraru et du wanimoc des chefferies de l'est. Un tel calendrier témoigne du fait que les vieux derniers-nés de cette chefferie ont définitivement cédé la parole au maître du mal ${ }^{10}$.

Le maître du mal évince les vieux derniers-nés et phagocyte le chef, ce qui produit donc "du roi" (doku). Mais si, comme nous l'avons vu, la parole des vieux derniers-nés est consubstantielle à l'igname, de quoi " se nourrit " alors celle des maîtres du mal ?

En frappant de mort un prestataire d'ignames, le kaze entame la personne des chefs " aînés des aînés " : au cours des puec, celle même du chef qu'il phagocyte; au cours des guerres, celle des autres chefs dont les prestataires d'ignames tués seront cannibalisés. Au-delà des limites des chefferies, les maîtres du mal ont ainsi partie liée dans le procès de réduction du " champ " agricole d'ordonnance sociale: ils transforment des prestataires d'ignames en viande de festin royal. Le "chef agricole" se rétrécit quand grandit le roi cannibale.

En apportant la guerre, qui compromet l'abondance exprimée par les fêtes des surplus wanimoc, le maître du mal s'engage à soumettre un plus grand nombre de * sujets " au roi, lequel verra converger vers lui un plus grand nombre de puec. Le roi est alors nommé doku hmaiai, « roi nombreux " (roi de nombreux sujets). Mais tout comme le meurtre

${ }^{9}$ Pour rendre compte de cet aspect bicéphale du * chef * nous emploierons désormais le terme * chef * (retok : “ aîné des aînés *) pour exprimer sa relation avec les vieux derniers-nés, et celui de * roi * $(d o k u)$ pour exprimer celle qu'il entretient avec le maître du mal.

10 Cet acania est le plus réputé du pays. Son action expansionniste sur plus de la moitié de l'île ne fut bloquée qu'en 1913 par l'administration française qui traça une ligne de délimitation $\mathrm{du}$ * district*, rétrocédant du territoire aux chefferies * spoliées *. 
de guerre, la coercition du puec va jusqu'à la production de cadavres comestibles. Cette chair humaine tient lieu et place de l'excédent wanimoc.

C'est donc toujours un élément constitutif d'un chef agricole qui est tué pour grandir un roi cannibale qui prolonge son intestin dans un maître du mal. Relevant de la vindicte du maître du mal, les victimes de son kaze et donc toute victime sont éviscérées sur-le-champ et les intestins, soigneusement vidés, lui seront servis avec le foie ${ }^{11}$. Un certain nombre de récits rapportent qu'on reconnaissait la violence des combats aux quantités d'excréments jonchant le champ de bataille. L'énergie du kaze (nen(e)) s'alimente de celle qui circule dans les intestins, car il faut, dit-on, « nourrir le kaze " (akodraruni kaze).

Dans la perspective du maître du mal et de son kaze, on voit donc que le meurtre cannibale ne consiste pas, à Maré, à répandre le sang mais les excréments de la victime ${ }^{12}$.

Mais alors, le maître du mal-souillé ne s'offre-t-il pas en déversoir au roi afin de prolonger en lui-même le transit intestinal de ce dernier ? C'est aussi sous la forme de chairs putrescentes, c'est-à-dire dejà " digérées" par leurs propres actions et transitant par la prothèse intestinale d'un roseau aspirateur, substitut de l'intestin du roi défunt, que le cadavre de ce dernier passe ensuite dans l'intestin qui lui survit. C'est au prix de n'être que l'intestin " siamois " du roi que le maître du mal partage l' " énergie " qui l'autorise à porter lui aussi le titre de "roi ".

On comprend alors qu'abandonnant les os du roi, même soigneusement dissimulés dans une grotte inconnue de tous, il les livre à cet autre maître du mal qui fait profession de découvrir de telles cachettes, lequel par manipulation de ces os de kaze saura pourvoir en chairs humaines, fraîches cette fois, un autre roi. C'est donc parce que le roi s'offre tout entier sous forme excrémentielle à l'appétit du maître du mal que ce

11 Le reste du corps est servi au roi doku qui le partage avec son entourage, voire avec tout le monde après les batailles " fertiles *.

12 Ce déplacement symbolique du sang vers les feces renvoie donc au déplacement de l'efficacité meurtrière : la magie de guerre des vieux derniers-nés, waceng, est supplantée par celle des maîtres du mal, kaze. 
dernier saura le pourvoir en chair frâiche. Ce cycle vient donc se substituer à celui qu'ouvrait la parole d'abondance agricole des vieux derniers-nés désormais muette.

Car, c'est selon la conception du cycle nutritionnel que s'exprime celui des prestations cérémonielles d'ignames. Si le chef (retok) en effet voit converger sur lui les prémices de l'ensemble de la production en l'espece d'ignames crues, il présentera lui-même un paquet dit ael ${ }^{13}$ contenant une igname entière cuite sans adjonction de lait de coco comme c'est le cas pour ce type de cuisson ${ }^{14}$. C'est donc une igname très sommairement cuisinée que l'aîné présente au dernier-né [sous ce rapport le "chef " (retok) au vieux dernier-né (eteshet)]. Or, lors du glissement d'autorité qui va du vieux dernier-né au maître du malsouillé, la prestation du souverain se transforme en excréments. Lorsque la bouche du vieux dernier-né reçoit de l'igname tout juste cuite, celle du maître du mal reçoit de l'igname digérée. Aux deux extrémités du procès nutritionnel sont situées les bouches respectives de l'un et de l'autre. L'opposition des substances fondée sur la seule transformation de l'igname induit ces bouches en paroles opposées ${ }^{15}$.

La souillure qui est ainsi placée sur le maître du mal est le pendant des meurtres des "sujets agricoles " constitutifs de la personne de " l'aîné des aînés ». Pour se débarrasser de cette souillure, le maître du mal n'aurait d'autre alternative, en toute logique, que d'occuper à son tour la place de la victime que l'on sert en festin au roi. Mais il faut, pour le percevoir, dégager davantage le meurtre cannibale de l'opacité du symbolisme plus général qui le comprend.

Les intestins font l'objet de soins nombreux et répétés chez les gens de Maré. Ainsi, avant la célébration des ignames nouvelles il n'est personne, des très petits enfants aux vieillards, qui ne se soumette à de

${ }^{13}$ Le ael est un large paquet de feuilles de bananiers ficelé de lianes, dans lequel on peut placer tous les aliments destinés à être cuits au four de pierres.

${ }^{14}$ Une telle présentation contraste avec celle requise habituellement pour une * bonne cuisine *. L'igname est coupée en tranches et baigne littéralement dans le lait de coco. Il en résulte une cuisson à l'étouffé qui rend le tubercule extrêmement tendre et savoureux.

15 Aussi l'igname est le * combustible * d'énergie, ce que vérifient les termes * cendres * $(c e-k o l)$ et \& feces * $(k o l)$ sous le même sémantème kol. 
longues purges d'eau de mer et de plantes. Il s'agit ainsi de « nettoyer » completement les intestins qui vont accueillir les nouvelles ignames. A cette même époque, on l'a vu, les poissons sont également "purgés " puisque leurs intestins sont nettoyés. Ainsi vidés, les poissons sont suspendus aux arbres pour que le vent emporte les dernières humeurs qui resteraient attachées à leurs chairs.

Tout porte à croire que cette précaution rituelle permet en fait de placer le poisson qui va accompagner l'igname nouvelle dans la position d'un oiseau. La vérification de cette hypothèse permettra d'établir qu'en menaçant les oiseaux, ou ce qu'on identifie à des oiseaux, on menace l'igname c'est-à-dire le cycle de prospérité agricole.

Le poisson, perché sur un arbre, est offert au vent purificateur parce qu'il doit accompagner l'igname nouvelle. Autrement dit tout être solidaire de l'igname " ressemble " à un oiseau. N'est-ce pas d'ailleurs ce qui ressort du nom porté par la fête des surplus agricoles : wanimoc, la fauvette ? A quelle nécessité symbolique répond une telle assimilation? Ce que le rituel révèle implicitement, le mythe en offre la confirmation explicite. Fondateurs de l'organisation sociale et du partage foncier, les mythes sur l'arrivée des ignames à Maré rapportent que lorsque ces dernières eurent accosté et qu'elles se furent avancées dans l'intérieur des terres, elles s'interrogèrent sur l'identité de leur nouveau pays. Le coq qu'elles avaient amené avec elles leur répondit de son cri : "Koko! Cerethi!" Cerethi est le nom du lieu où les ignames se trouvaient rendues $^{16}$; koko, le cri onomatopéique du coq, est le nom même qui signifie «igname ". La parole du coq informe les ignames parce que l'igname est parole de coq.

Le coq, nous l'avons vu, est un symbole du chef. Comme celui-ci, le coq doit déléguer sa parole à un dernier-né, qui n'est autre, dans le monde des volatiles, que la fauvette wanimoc dont la fête des surplus agricoles porte le nom. Les vieux derniers-nés, on s'en rappelle, ont seuls pouvoir de décréter cette fête.

${ }^{16}$ Par extension, c'est une grande partie du littoral est de l'île que l'on nomme aussi Cerethi. 
Souvenons-nous à présent que la chair humaine issue du puec ou de la guerre tient lieu et place des surplus wanimoc et, par ailleurs, que cette chair humaine requiert l'efficience d'un os-de-kaze également nommé os-de-coq. Le mythe recouvre ici parfaitement la pratique sociale. Si le roi-coq, délégant sa parole au dernier-né-fauvette, assure l'abondance des ignames, il la confie d'autres fois à un tueur d'hommes qui, muni d'un os-de-coq, le pourvoit en chair humaine. De cela, il ressort aussi l'identification rituelle et mythique de la chair humaine à de la chair d'oiseau ${ }^{17}$. Le kaze, soit l'os-de-coq, ne demande-t-il pas à être « nourri " de chair humaine ?

C'est par référence symbolique au monde des volatiles que se dit le meurtre cannibale. Nous avons noté aussi que le rituel n'hésite pas à utiliser un poisson comme s'il se fût agi d'un oiseau. C'est que, sans doute, « compagnon * de l'igname nouvelle, le poisson reçoit ainsi quelque chose de l'attribut sonore du coq. Mais, s'il est nécessaire de tirer des « chairs » du monde aquatique ${ }^{18}$, n'est-ce pas en même temps pour évoquer une autre instance articulée selon l'opposition poisson/volatile?

Dans leur étroite relation, maître du mal et roi disent être comme " la poule et le coq ". Ils se réferent ainsi à une relation matrimoniale qui, sous l'angle rhétorique du maître du mal, consiste à dire que la poule épouse le coq dont elle sait manipuler les os. Nous reviendrons sur ce point après avoir rappelé brievement les caractéristiques symboliques de la nomenclature de l'échange matrimonial.

${ }^{17}$ Le mythe de la fauvette wanimoc aurait pu suffire à cette démonstration. Adrapo, la mante religieuse, pleure la perte de la conque (que l'on fait sonner pour réunir le monde). C'est l'ogre Kazenir qui la lui a prise. Aucun volatile n'a le courage d'aller la récupérer dans l'antre de l'ogre ; seul Wanimoc, le dernier-né des volatiles, ose s'y aventurer et réussit à récupérer l'instrument sonore. On voit donc que les volatiles craignent d'être mangés par Kazenir dont on reconnaît au passage le kaze qui en forme le nom, c'est-à-dire d'être traités comme les hommes dont Kazenir apprécie la chair. Ce cannibale a fait main basse sur l'instrument qui rassemble le peuple, la parole de l'aîné des aînés que le dernier-né peut restituer. Cette parole est donc alternative : ou celle du Kaze(-nir) et la chair humaine, ou celle du dernier-né comme transmission du cri du coq * l'igname *.

${ }^{18}$ La locution lae ia * pêcher * signifie littéralement * acquérir (de la) chair, (de la) viande $»$. 
Le mariage avec la cousine croisée matrilatérale procède d'un échange de sceurs qui, répété à la deuxième génération, prend l'aspect d'un mariage avec la cousine bilatérale. Or, l'échange des sœurs se dit en terme de " chairs ", ia. Au sens littéral de l'énoncé vernaculaire, le frère qui cède sa sceur se met "à l'affût " de celle qu'un principe immanent de réciprocité l'autorise à attendre. "Beau-frère" se dit rabaian, "être à l'affût, épier la chair " (raba-ia-n). Celui qui cède la chair de sa sæur est à l'affût de celle qui doit lui être cédée. Or, cette chair de saur qu'il cède à un tiers, et dont le retour est attendu sous l'espèce de la sœur du " beau-frère ", est métaphoriquement identifiée a de la chair de poisson. La terminologie de la pêche lève toute incertitude sur la métaphore matrimoniale prégnante dans les mythes et la célébration des prémices. En effet, "pêcher le gros poisson à la ligne de traîne " est rendu par la locution raba ia. "Guetter ", raban, est le radical de "beau-frère ". Raba ia : "guetter le poisson à la ligne de traîne " n'est autre que le mot « beau-frère " lui-même. De cette façon l'expression lae hmenew, "acquérir, prendre femme », glisse et se plaque sur lae ia, mot à mot "acquérir viande, chair " c'est-à-dire "pêcher ". Les beaux-frères "pêchent" donc réciproquement la « chair» de leur sœur.

Il faut enfin, pour valider la perspective aquatique du preneur d'épouse, faire état d'un second volet dans l'énoncé du théorème symbolique de l'échange des sæurs. Si le preneur reçoit la chair aquatique d'une épouse, il cède en retour la chair aérienne d'une sour. L'analyse du corpus mythique permet de dégager, par récurrences multiples, le modele symbolique invariant de l'échange des soeurs par réversibilité des mondes marin et aérien à l'interface desquels opèrent les "guetteurs-de-chairs": la chair qui est reçue comme du poisson (cousine matrilatérale) fut cédée comme chair de volatile (sceur), et vice versa.

Cette chair (ia), prohibée entre frère et sœur, est donc sans aucun doute possible de la chair de volatile. $\mathrm{Si}$, comme on l'a montré plus haut, le cannibalisme opère symboliquement sur la chair des volatiles, on vérifie à Maré, ce qui fut observé en maints autres lieux, que l'inceste est compris comme une forme de cannibalisme. 
Est-ce à dire alors que le roi et son maître du mal, avouant une relation entre poule et coq, révèlent la nature incestueuse de l'échange matrimonial qui les lie ? Comme on s'en souvient, le paradigme complet est que la poule épouse le coq dont elle sait manipuler les os. Contrairement à l'échange matrilatéral réalisé par des hommes à l'« affût de chairs aquatiques », il s'agit à présent d'un « détournement d'os de volatile " dont l'épouse (la poule) a l'initiative. Les chairs de volatile ne sont donc pas mis en cause et le type de mariage dont il est fait état échappe, de justesse semble-t-il, à l'inceste. Si, comme pour des obsèques, la procédure matrimoniale requiert la tutelle des maternels pour permettre la circulation de l'énergie du nen(e), on comprend que lorsqu'une femme est initiatrice de l'échange (la poule manipule les os du coq) elle épouse son cousin matrilatéral pour lequel elle est une cousine patrilatérale. De cette inversion des sexes dans l'initiative de l'échange dépend l'inversion symbolique de l'acquisition de chair aquatique (matilatérale) en os de volatile (patrilatérale). Se dégage ainsi une alliance qui permet aux maîtres du mal de toute l'île de " gérer » la guerre dans un continuum (une chaîne d'alliance patrilaterale) qui joue sur l'opposition apparente des chefferies.

On découvre ainsi la place inattendue de la sceur du maître du mal, epouse du roi ${ }^{19}$, laquelle, manipulatrice subsumee des os de kaze d'un autre roi défunt, réclame d'être nourrie de cette chair humaine dont on pourvoit son royal époux ${ }^{20}$. Derrière le maître du mal se tenait une femme tout comme, nous l'avions noté, les vieux derniers-nés portent en fait le nom de "vieilles ", eteshet. Cette duplicité des sexes ressurgira ailleurs.

19 Du moins pour les premières générations à compter de l'introduction du maître du mal dans la chefferie.

20 Ta'unga, teacher rarotongan de la London Missionary Society, rapporte dans le récit de son séjour en Nouvelle-Calédonie et aux îles Loyauté, bien avant l'occupation coloniale, qu'il y avait une princesse cannibale à Maré : * Ta'unga l'attendit et vit avec horreur tous les crânes de tous ceux qu'elle avait dévorés, autour de son habitation. Il demanda qui avait mangé tous ces hommes. Elle répliqua que c'était elle. Quand il exprima sa surprise à cette vue elle répondit : "Oh, il n'y a pas de nourriture si agréable et savoureuse que la chair humaine" (Pisier, 1980). 
Pour forger un instrument meurtrier qui offrira à sa sæur la chair qui lui fut soustraite lors d'un mariage d'os, le maître du mal se présente à la souillure du roi en le prolongeant de son propre intestin afin de faire circuler l'énergie utérine nen(e) compromise par un mariage prétendu matrilatéral. Mais comment cette chair est-elle identifiee à celle de l'inceste?

L'expression $d u$-re-kaze " os-de-cadavre " s'oppose par inversion symétrique à celle de du-re-hna-hnapo «os-de-naissance ${ }^{21}$.

Le du-re-hna-hnapo " os-de-naissance " est, du point de vue de la sœur, la part d'enfants due au frère. La filiation matrilinéaire est perçue comme patrilinéaire selon la perspective de la sœur ; c'est pourquoi elle s'exprime en terme d'os. Cet os est porteur de chair puisqu'apparu lors du procès de naissance.

Le $d u$-re-kaze « os-de-mort » est cette part d'os, mais dépourvue de chair parce que mort, rendue à la génération suivante par l'oncle utérin, soit un cousin matrilatéral du point de vue de l'épouse. Le mariage patrilatéral est perçu cette fois comme matrilatéral selon la perspective de la saur.

Les « os-de-naissance » remontent vers les oncles utérins (filiation, pour une part), les « os-de-mort " descendent vers les nièces utérines (mariage, patrilatéral seulement).

On a vu que cet os-de-kaze est l'instrument meurtrier qui permet de pourvoir l'épouse en chair humaine. Cette dernière reconstitue ainsi la chair que pourront partager les frères et sœurs qu'elle mettra au monde. Le meurtre cannibale permet donc de combler un vide de chair issu de l'alliance imposée par les maîtres du mal. Encore faut-il totalement boucler le circuit de chair qui passe des sœur et frère à l'oncle utérin et qui s'interrompt à l'époux d'os, cousin matrilatéral. La

${ }^{21}$ Le du-re-hna-hnapo * os-de-ce-qui-est-né * est l'enfant ou les enfants, garçon ou fille, ainé ou cadet, selon l'accord (ekonejew) passé au cours des transactions de mariage (ekon), qui sera ou seront attribués au frère, ou aux frères, de l'épouse. La notion de * point de vue * dans la reproduction des rapports de parenté semble si importante, qu'il nous paraît indispensable d'aborder l'analyse de la parenté en renonçant dès le départ au langage théorique classique pour cerner le vaste ensemble métaphorique à partir duquel opère la réflexion locale. Sous cet angle, c'est toute une description analytique de la parenté qui reste à faire. 
connexion intestinale entre ce dernier et le frere le permettra, mais au prix d'une symbiose qui, du point de vue de la sceur, rapporte le frère à l'époux et marque cette alliance cannibale du sceau de l'inceste.

En devenant l'intestin siamois du roi, le maître du mal brouille la perception des épouses qui président à l'échange des os et qui ne peuvent plus établir de discrimination entre époux d'os et frère de chair. Le maître du mal induit ainsi un « doute de chair " tout en offrant celle des victimes de l'os. Le cannibalisme exprime ainsi l'inceste reproducteur de chairs issu de l'impossible discrimination des chairs à laquelle auvre la complexité de l'institution politique: l'autorité masculine fondée du point de vue des femmes.

Le brouillage de la discrimination des chairs se traduit symboliquement par le décloisonnement des espaces séparés aquatique et aérien où se maintient « normalement " l'opposition des chairs matrimoniales. La chair de l'inceste circule alors sans entrave, sans interdit, entre ces espaces. Le sacrifice de la tortue permettra de réintroduire la discrimination des chairs.

Lorsqu'une tortue est capturée, il appartient au maître du rivage concerné de la porter à la chefferie où elle sera mise à mort par le maître du mal et partagée par le vieux dernier-né entre le roi et ceux de son entourage. Le maître du mal-souillé en reçoit la tête. Le sort particulier qui est réservé à la tortue s'explique par le fait qu'elle occupe une place indéterminée dans la taxinomie animale. Elle vit à l'origine, selon le mythe, sur le plateau de l'île, accompagnée de l'oiseau " collier blanc " mekece (Columba hypoenochroa). Ce dernier lui permit un jour de voir le pays à partir du ciel. Tortue et collier blanc jouent un autre jour à cache-cache. Tortue découvre collier blanc perché sur un arbre parce qu'elle aperçoit son reflet dans une flaque d'eau. Mauvais perdant, le collier blanc défèque sur la tête de la tortue. Elle descend donc au bord de mer pour se laver mais la honte la pousse à élire domicile dans la mer d'où elle ne rejoint désormais le rivage que pour pondre.

«Tortue » se dit cewen. Ce mot se présente comme métathèse de cenew « jeune fille». Ce rapport lexical est vérifié par le fait que cewen est la tortue femelle. Le mâle uac(e), soit $u$-ac(e) "pénis-chose ", est lexicalement surdéterminé en tant que mâle. «L'anagramme de la 
tortue " surdétermine en tant que jeune fille cet être que l'on apporte au roi et que l'on nomme cewen même s'il s'agit d'un mâle dont on paraît ignorer le sexe. Encore une fois la duplicité des sexes se fait jour. Associée aux êtres aériens (qui jettent leur " reflet " sur la surface des eaux), la souillure de l'excrément place la tortue dans l'espace aquatique qui est un réservoir d'épouses ${ }^{22}$. Le "guetteur de chairs " en sera donc trompé. Ses chairs, qui transgressent l'étanchéité réciproque des espaces aérien et aquatique, expriment la corruption des règles de l'échange. La discrimination des chairs matrimoniales est rendue alors grâce au partage sacrificiel des vieux derniers-nés. Ces derniers reprennent la parole et restaurent la perspective des " vieilles " dont ils portent le nom, perspective qui appréhende, sous la détermination anagrammatique de « jeune fille », le maître du mal à la tête souillée.

Charles Illouz Université de La Rochelle

\section{Références bibliographiques}

M. Godelier

1982 La production des Grands Hommes, Paris, Fayard.

B. Juillerat

1986 Les Enfants du sang, Paris, Maison des Sciences de l'Homme.

G. Pisier

1980 Le témoignage de Ta'unga, Société d'études historiques de la nouvelle-Calédonie, $n^{\circ} 25$, Nouméa.

22 On observe à nouveau un déplacement du sang vers les feces dans le rite de purification des jeunes filles lors de leurs premières règles. Elles sont menées, comme la tortue, au bord de mer où on s'occupera, non du sang des règles, mais encore de leurs intestins. Longuement purgées d'eau de mer et de plantes, elles sont libérées de ce qui pourrait nuire à la * vie trouvée *, hna uni ciroi, expression désignant la menstruation. 